Archive for

Organic Chemistry

Arkivoc 2018, part vi, 0-0

\title{
Isoparametricity and related phenomena in reactions of trans-2,3-diaryloxiranes with arenesulfonic acids
}

\author{
Igor V. Shpan'ko*a and Irina V. Sadovaya ${ }^{b}$ \\ ${ }^{a}$ Educational-Scientific Institute of Chemistry, Vasyl' Stus Donetsk National University, \\ 21600 Richchia Street, 21021 Vinnytsia, Ukraine \\ ${ }^{b}$ Department of Chemistry, Donetsk National University, 24 Universitetskaya Street, 83001 Donetsk, Ukraine \\ Email: shpanko16@ukr.net
}

Received 03-05-2019

Accepted 07-24-2019

Published on line 09-02-2019

\section{Abstract}

This article is a continuation of a series of publications devoted to the systematic study of the still poorly investigated unique isoparametricity phenomenon in chemistry. Quantitative evidence for all variants of the second-order interaction structure-structure and structure-temperature types were obtained in the reactions between X-substituted trans-2,3-diaryloxiranes and Y-substituted arenesulfonic acids. Experimental evidence for the reality of isoparametric points with respect to the parameters of variable factors was obtained. Of particular importance is the proof of the physical reality of such an aspect of isoparametricity as the widely discussed enthalpy-entropy compensation effect. Important information was obtained about the intriguing properties of the three-parameter relationships with cross terms, concerning such attributes as the critical values of the parameters of the variable factors. At these critical values corresponding terms in threeparameter regressions disappear.

$$
\begin{aligned}
& \mathrm{XC}_{6} \mathrm{H}_{4(3)} \cdot{ }_{\mathrm{O}}^{\mathrm{C}_{6} \mathrm{H}_{4(3)} \mathrm{X}}+\mathrm{YC}_{6} \mathrm{H}_{4} \mathrm{SO}_{3} \mathrm{H} \stackrel{T}{\longrightarrow} \text { Products } \\
& T=265,281,298 \mathrm{~K} \\
& \mathrm{X}=\mathrm{H}, 4-\mathrm{Br}, 4-\mathrm{NO}_{2}, 3-\mathrm{Br}-5-\mathrm{NO}_{2} ; \mathrm{Y}=4-\mathrm{OMe}, 4-\mathrm{Me}, \mathrm{H}, 4-\mathrm{Cl}, 3-\mathrm{NO}_{2}
\end{aligned}
$$

Keywords: Oxirane ring opening, structure-reactivity relationships, isoparametricity, activation parameters, compensation effect 


\section{Introduction}

Efficient control of chemical processes requires knowledge of the quantitative relations describing the effects of various internal and external factors (structure, solvent, temperature, $\mathrm{pH}$ of medium, pressure, etc.) on their kinetic, activation, thermodynamic, and other characteristics. This fundamental problem common to the whole of chemistry has been generally solved in terms of correlation analysis. Many various empirical relationships have been widely used to estimate the influence of the different factors on organic reactivity. ${ }^{1-7}$ They demonstrate the surprising universality of the principle of linearity of free energies, which Palm has shown is a particular case of the polylinearity principle. ${ }^{7}$ Of special interest are polylinear equations (PLEs) with cross terms, taking into account non-additivity (interaction) of the effects of mutually varied factors. This makes them uniquely suitable for the analysis of chemical data. Predictive capabilities of PLEs are significantly improved compared to traditional one-parameter correlations. The presence of cross terms is implied by the general form of PLEs ${ }^{7}$ and is interpreted as a change in the intensity of the effect of one factor under the influence of another factor. Non-additivity of the effects of two cross-varied factors determines such an intriguing property of PLEs as isoparametricity. ${ }^{7,8}$ This term means that the coefficient of sensitivity to the effect of one of factors in one-parameter correlations becomes zero for the special value of the parameter of another factor called isoparametric point (IPP). As a real phenomenon, isoparametricity is a challenge to traditional concepts in chemistry. Despite this, little attention is paid to its study. One of the reasons is that the IPPs in many processes fall within the area of distant extrapolation and are unattainable experimentally. In this context, the experimental evidence of isoparametricity is an actual phenomenological task. Our previous studies have shown that nucleophilic substitution reactions at carbonyl, benzyl, and benzhydryl carbon atoms, ${ }^{9-12}$ along with nucleophilic reactions of oxirane ring opening, ${ }^{13-18}$ are very promising for the experimental investigation of isoparametricity in all its aspects. The systematic study of multifactor effects on the kinetics of these reactions revealed a wide variety of IPPs in cross correlations of the structure-structure, structure-solvent, structure-temperature, and solvent-temperature types. Some of these points were passed through, which is a rare situation in chemical processes, and this was accompanied by reversal of the signs of the corresponding sensitivity coefficients because of reactivity reorientation (isoparametricity paradox).

It should be noted that the oxirane ring opening reactions are of particular interest because they clearly demonstrate such a special aspect of isoparametricity as an enthalpy-entropy compensation effect (EECE), which has been the subject of hot debate by scientists for many decades..$^{19-33}$ Earlier, ${ }^{16-18}$ we have studied the joint effects of structure and temperature on the rates and activation parameters of the reactions of symmetrically $\mathrm{X}$-substituted trans-2,3-diaryloxiranes (1a-d), $\mathrm{X}=\mathrm{H}$ (1a), 3- $\mathrm{Br}(\mathbf{1 b}), 4-\mathrm{NO}_{2}$ (1c), 3- $\mathrm{Br}-5-\mathrm{NO}_{2}$ (1d) with $\mathrm{Y}$-substituted arenesulfonic acids $(\mathbf{2 a}-\mathrm{e}), \mathrm{Y}=4-\mathrm{OCH}_{3}(\mathbf{2 a}), 4-\mathrm{CH}_{3}(\mathbf{2 b}), \mathrm{H}(\mathbf{2 c}), 4-\mathrm{Cl}(\mathbf{2 d}), 3-\mathrm{NO}_{2}(\mathbf{2 e})$ in a dioxane-1,2-dichloroethane mixture (7:3/v:v) at 265, 281, and $298 \mathrm{~K}$.

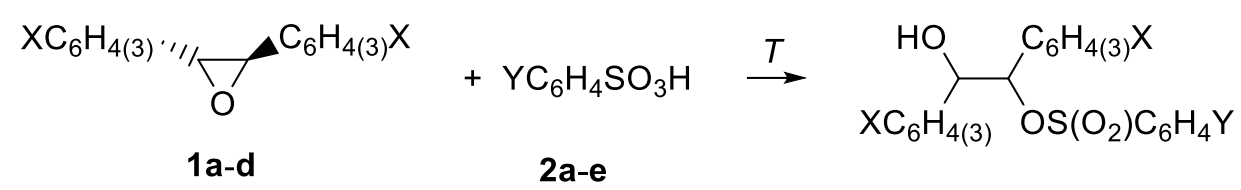

Scheme 1. Reactions of oxiranes 1a-d with arenesulfonic acids $\mathbf{2 a - e .}$

Since in the reaction series three factors were cross-varied (substituents $X, Y$, and temperature $T$ ), this allowed us to test the manifestation of various combinations of the second-order interactions of their effects, as well as the third-order interaction. A part of the results of cross correlation analysis of a kinetic experiment 
was presented in the publications mentioned above. Here we report summarizing information about the study of reactions of oxiranes $\mathbf{1 a}$ - $\mathbf{d}$ with acids $\mathbf{2 a - e}$ in multifactorial conditions.

\section{Results and Discussion}

To quantify the effects of the cross-varied factors on the rate and free activation energy of reactions shown in Scheme, their kinetic and activation parameters taken from recent papers ${ }^{16-18}$ were subjected to a regression analysis.

\section{Two-parameter correlations}

To evaluate the effects of two-variable factors $i$ and $j$ at a fixed parameter of the third factor $h$, on the reaction rate the following PLE was used

$$
\log k_{\mathrm{ijh}}=\log k_{00 \mathrm{~h}}+q_{\mathrm{i}}{ }^{\mathrm{Oh}} \mathrm{x}_{\mathrm{i}}+q_{\mathrm{j}}{ }^{\mathrm{Oh}} \mathrm{x}_{\mathrm{j}}+q_{\mathrm{ij}} \mathrm{h}_{\mathrm{i}} \mathrm{x}_{\mathrm{j}}
$$

Here $\log k_{00 h}$ is the value of $\log k_{\mathrm{ijh}}$ at randomly selected standard values of the parameters of the factors $i$ and $j$, e.g., $x_{i}=0$ and $x_{j}=0, q_{i}{ }^{\text {hh }}$ and $q_{j}{ }^{\text {hh }}$ are coefficients of the sensitivity toward $x_{i}$ and $x_{j}$ under standard conditions $\left(x_{j}=0\right.$, and $x_{i}=0$, respectively), $q_{i j}{ }^{h}$ is the cross-interaction coefficient, which reflects the perturbing (nonadditive) effects of factors $i$ and $j$ at a fixed factor $h$ (in this and subsequent equations, the subscripts and superscripts relate respectively to the varied and fixed factors). The presence of a cross term $q_{i j}{ }^{\mathrm{h}}$ in PLE 1 enables to calculate two IPPs: $x_{i j(j}{ }^{h}=-q_{j}{ }^{0 h}\left(q_{i j}{ }^{h}\right)^{-1}$ and $x_{j(i)}{ }^{h}=-q_{i}{ }^{0 h}\left(q_{i j}{ }^{h}\right)^{-1}$ (hereinafter, the subscript in parentheses refers to the second variable factor). At these IPPs the magnitude of log $k_{\mathrm{ijh}}$ is the same, i. e. $\log k_{\mathrm{ijh}}=\log k_{00 \mathrm{~h}}-$ $q_{\mathrm{i}}{ }^{\mathrm{Oh}} q_{\mathrm{j}}{ }^{\mathrm{Oh}}\left(q_{\mathrm{ij}}{ }^{\mathrm{h}}\right)^{-1}$, and remains constant when either the factor $j$ at the IPP $\mathrm{x}_{\mathrm{i}(\mathrm{j})}{ }^{\mathrm{h}}\left(q_{\mathrm{j}}^{\mathrm{ih}}=0\right)$, or the factor $i$ at the IPP $\mathrm{x}_{\mathrm{j}(\mathrm{i})}{ }^{\mathrm{h}}\left(q_{\mathrm{i}}^{\mathrm{jh}}=0\right)$, is varied.

The PLE 1 has the form of PLEs 2-4, describing the combined effects of substituents $X$ and $Y$ at a fixed temperature $T$, substituents $Y$ and $T$ with a fixed substituent $X$, and substituents $X$ and $T$ with a fixed substituent $\mathrm{Y}$.

$$
\begin{aligned}
& \log k_{\mathrm{XYT}}=\log k_{00 \mathrm{~T}}+q_{\mathrm{X}}{ }^{0 \mathrm{~T}} \tau_{\mathrm{X}}+q_{\mathrm{Y}}{ }^{0 \mathrm{~T}} \sigma_{\mathrm{Y}}+q_{\mathrm{XY}}{ }^{\top} \tau_{\mathrm{X}} \sigma_{\mathrm{Y}} \\
& \log k_{\mathrm{XYT}}=\log k_{\mathrm{X} O \mathrm{~T}}=\infty+q_{\mathrm{Y}}{ }^{\mathrm{XT}={ }^{\infty}} \sigma_{\mathrm{Y}}+q_{\mathrm{T}}{ }^{\mathrm{X}} 1000 / T+q_{\mathrm{YT}}{ }^{\mathrm{X}} \sigma_{\mathrm{Y}} 1000 / T \\
& \log k_{\mathrm{XYT}}=\log k_{\mathrm{OYT}=\infty}+q_{\mathrm{X}}{ }^{\mathrm{YT}}={ }^{\infty} \tau_{\mathrm{X}}+q_{\mathrm{T}}{ }^{0 \mathrm{Y}} 1000 / T+q_{\mathrm{XT}}{ }^{\mathrm{Y}} \tau_{\mathrm{X}} 1000 / T
\end{aligned}
$$

Here $\sigma_{Y}$ is the Hammett constant for substituent $Y$ and $\tau_{X}=\log k_{00 T}-\log k_{\text {XоT }}(T=265 \mathrm{~K})$ is a quantitative characteristic of total effects of substituents $X$ ( $\tau_{x}$ for $X$ in oxiranes $\mathbf{1 a}, \mathbf{1 b}, \mathbf{1 c}$, and $\mathbf{1 d}$ are equal to $0,2.02,3.47$, and 4.38 , respectively ${ }^{15}$ ). The values of the coefficients of PLEs 2-4 calculated for various two-factor crossreaction series with the use of kinetic data ${ }^{17}$ are given in Table 1.

Statistical significance of the cross-interaction coefficients $q_{\mathrm{XY}}{ }^{\top}, q_{\mathrm{YT}}{ }^{\mathrm{X}}$, and $q_{\mathrm{XT}}{ }^{\mathrm{Y}}$ show that all variants of the second-order interactions of the effects of the substituents $X, Y$ and temperature $T$ should be considered when assessing the reactivity of the studied reaction system. At the same time, the invariance of these coefficients when the parameters of fixed factors are changed indicates the absence of third-order interaction $\left(q_{\mathrm{XYT}}=0\right)$. 
Table 1. The coefficients of PLEs 2-4 ( $\left.R^{a} \geq 0.995\right)$ and IPPs for the two-factor cross-reaction series including reactions of oxiranes $1 \mathrm{a}-\mathbf{d}$ with acids $\mathbf{2 a - d}$ in the dioxane-1,2-dichloroethane mixture (7:3/v:v) at 265, 281, and $298 \mathrm{~K}$

\begin{tabular}{|c|c|c|c|c|c|c|c|}
\hline Fixed factor $h$ & $\log k_{00 h}$ & $q_{i}{ }^{\text {oh }}$ & $q_{\mathrm{j}}^{\mathrm{oh}}$ & $q_{i j}{ }^{h}$ & $x_{i(j)^{h}}$ & $x_{j(i)^{h}}$ & Refs. \\
\hline$T$ & $\log k_{00 T}$ & $q x^{0 T}$ & $q_{\mathrm{Y}}{ }^{0 \mathrm{~T}}$ & $q_{X Y}^{\top}$ & $\tau_{X(Y)}{ }^{\top}$ & $\sigma_{Y(X)}{ }^{\top}$ & \\
\hline $265 \mathrm{~K}$ & $-1.24 \pm 0.01$ & $-1.00 \pm 0.01$ & $1.61 \pm 0.04$ & $-0.34 \pm 0.01$ & $4.73^{b}$ & -2.94 & 15 \\
\hline $281 \mathrm{~K}$ & $-0.79 \pm 0.04$ & $-0.90 \pm 0.01$ & $2.01 \pm 0.02$ & $-0.33 \pm 0.06$ & 6.09 & -2.73 & c \\
\hline $298 K$ & $-0.46 \pm 0.05$ & $-0.76 \pm 0.01$ & $2.50 \pm 0.03$ & $-0.34 \pm 0.08$ & 7.35 & -2.23 & c \\
\hline$x$ & $\log k_{\mathrm{X} O T}=\infty$ & $q_{\mathrm{Y}} \mathrm{XT}=\infty$ & $q_{\mathrm{T}}{ }^{\mathrm{X} 0}$ & $q_{\mathrm{YT}} \mathrm{x}$ & $\sigma_{Y(T)} x$ & $T_{(\mathrm{Y})} \mathrm{X} / \mathrm{K}$ & \\
\hline $\mathrm{H}$ & $6.2 \pm 0.3$ & $10.1 \pm 0.9$ & $-1.96 \pm 0.07$ & $-2.2 \pm 0.4$ & -0.89 & 220 & c \\
\hline $4-\mathrm{NO}_{2}$ & $8.5 \pm 0.3$ & $8.3 \pm 0.9$ & $-3.52 \pm 0.09$ & $-2.0 \pm 0.2$ & -1.76 & 241 & 16 \\
\hline $3-\mathrm{Br}-5-\mathrm{NO}_{2}$ & $11.7 \pm 0.1$ & $8.4 \pm 0.7$ & $-4.61 \pm 0.04$ & $-2.2 \pm 0.2$ & -2.09 & $262^{b}$ & 18 \\
\hline$Y$ & $\log k_{\mathrm{OYT}}=\infty$ & $q_{\mathrm{X}}^{\mathrm{YT}=\infty}$ & $q_{T^{O Y}}$ & $q_{\mathrm{XT}}{ }^{\mathrm{Y}}$ & $\tau_{X(T)}{ }^{Y}$ & $T_{(\mathrm{X})}{ }^{\mathrm{Y}} / \mathrm{K}$ & \\
\hline $4-\mathrm{OCH}_{3}$ & $3.1 \pm 0.3$ & $1.1 \pm 0.4$ & $-1.4 \pm 0.3$ & $-0.51 \pm 0.09$ & -2.74 & 464 & c \\
\hline $4-\mathrm{CH}_{3}$ & $4.0 \pm 0.5$ & $1.2 \pm .0 .3$ & $-1.5 \pm 0.3$ & $-0.60 \pm 0.09$ & -2.50 & 500 & c \\
\hline $\mathrm{H}$ & $5.0 \pm 0.4$ & $1.3 \pm 0.4$ & $-1.8 \pm 0.3$ & $-0.61 \pm 0.08$ & -2.95 & 469 & c \\
\hline $4-\mathrm{Cl}$ & 8.3. \pm 0.7 & $1.0 \pm 0.2$ & $-2.4 \pm 0.2$ & $-0.55 \pm 0.07$ & -4.36 & 550 & c \\
\hline
\end{tabular}

${ }^{a}$ A multiple correlation coefficient. ${ }^{b}$ Experimentally observed IPP. ${ }^{c}$ This work.

Of the twenty calculated IPPS, such as $\tau_{X(Y)}{ }^{\top}, \tau_{X(T)}{ }^{Y}, \sigma_{Y(X)}{ }^{T}, \sigma_{Y(T)}{ }^{X}, T_{(Y)}{ }^{X}$, and $T_{(X)}{ }^{Y}$, only two points proved to be experimentally achievable. As can be seen from Table 1, in the cross-reaction series with variable substituents $\mathrm{Y}$ and temperature $T$ at the fixed substituent $\mathrm{X}=3-\mathrm{Br}-5-\mathrm{NO}_{2}$ in oxirane $1 \mathrm{~d}$ the IPP $T_{(\mathrm{Y})}{ }^{\mathrm{X}}=262 \mathrm{~K}$ is close to a temperature of $265 \mathrm{~K}$ in an experiment. In accordance with the regularities of isoparametric dependencies at this IPP substituents $Y$ in the acidic reagent should not influence on the rate of these reactions $\left(q_{Y}{ }^{X T}=0\right)$. This is illustrated by a decrease in the sensitivity coefficient $q_{\mathrm{Y}}{ }^{\mathrm{XT}}$ to the effects of the substituents $\mathrm{Y}$ practically to zero in the reactions of oxirane $1 \mathbf{d}$ with a decrease in temperature: ${ }^{17} q_{\mathrm{Y}}{ }^{\mathrm{XT}}(T \mathrm{~K})=1.01 \pm 0.09(298 \mathrm{~K}), 0.50 \pm 0.04$ (281 K), $0.10 \pm 0.05$ (265 K).

The IPP $\tau_{X(Y)}{ }^{\top}=4.73$ was almost reached in the reaction series with variable substituents $X$ and $Y$ at a fixed temperature of $265 \mathrm{~K}$, since the value $\tau_{X}=4.38$ for the substituent $X$ in oxirane $1 \mathbf{d}$ is close to the value of this IPP. At this point the effects of the substituents $Y$ should not appear, which is confirmed by a decrease in $q_{\mathrm{Y}} \mathrm{XT}^{\mathrm{T}}$ $(T=265 \mathrm{~K})=1.57 \pm 0.02,1.00 \pm 0.04,1.50 \pm 0.03,0.10 \pm 0.05$ with an increase in the electron-withdrawing properties of the substituents $X$ in oxiranes $\mathbf{1 a}, \mathbf{1 b}, \mathbf{1 c}$, and $\mathbf{1 d} .{ }^{17}$

In terms of activation parameters, the second-order interactions of the effects of structure and temperature can be described by a PLE:

$$
\Delta G_{i j h}{ }^{\neq}=\Delta G_{00 h}{ }^{\neq}+Q_{i}{ }^{0 h} x_{i}+Q_{j}{ }^{0 h} x_{j}+Q_{i j}{ }^{h} x_{i} x_{j}
$$

Here $\Delta G_{00 h}{ }^{*}$ is the free energy of activation under standard conditions $\left(x_{i}=x_{j}=0\right), Q_{i}{ }^{0 h}$ and $Q_{j}{ }^{\text {oh }}$ are parameters of the standard reactions at $x_{j}=0$ and $x_{i}=0$, respectively, $Q_{i j}{ }^{h}$ is the cross-interaction coefficient at a fixed factor $h$. The PLE 5 has the form of PLEs 6-8, describing the combined effects of substituents $X$ and $Y$ at a fixed temperature $T$, substituents $Y$ and $T$ with a fixed substituent $X$, and substituents $X$ and $T$ with a fixed substituent $\mathrm{Y}$. 


$$
\begin{aligned}
& \Delta G_{\mathrm{XYT}}{ }^{\neq}=\Delta G_{00 \mathrm{~T}^{*}}+Q_{\mathrm{X}}{ }^{0 \mathrm{~T}} \tau_{\mathrm{X}}+Q_{Y}{ }^{0 \mathrm{~T}} \sigma_{Y}+Q_{X Y}{ }^{\top} \tau_{X} \sigma_{Y} \\
& \Delta G_{\mathrm{XYT}}{ }^{\neq}=\Delta G_{\mathrm{X} O 0^{\neq}}+Q_{\mathrm{Y}}{ }^{\mathrm{X} 0} \sigma_{\mathrm{Y}}+Q_{\mathrm{T}}{ }^{\mathrm{X} 0} T+Q_{\mathrm{YT}^{\mathrm{X}}}{ }^{\mathrm{X}}{ }_{\mathrm{Y} T} \\
& \Delta G_{\mathrm{XYT}}{ }^{\neq}=\Delta G_{\mathrm{OYO}^{*}}{ }^{\neq}+Q_{\mathrm{X}}{ }^{\mathrm{YO}} \tau_{\mathrm{X}}+Q_{\mathrm{T}^{\mathrm{O}}} T+Q_{\mathrm{XT}}{ }^{\mathrm{Y}} \tau_{\mathrm{X}} T
\end{aligned}
$$

The coefficients of PLEs 6-8 calculated for various two-factor cross-reaction series with the use of the $\Delta G_{\mathrm{XYT}}{ }^{\neq}$ values taken from our previous work ${ }^{17}$ are presented in Table 2.

Table 2. The coefficients of PLEs 6-8 $(R \geq 0.985)$ and IPPs for the two-factor cross-reaction series including

\begin{tabular}{|c|c|c|c|c|c|c|c|}
\hline Fixed factor $h$ & $\Delta G_{00 h^{*}}$ & $Q_{i}^{0 h}$ & $Q_{\mathrm{j}}^{\text {oh }}$ & $Q_{i j}^{\mathrm{h}}$ & $x_{i(j)}^{h(G)}$ & $X_{j(i)}{ }^{h(G)}$ & Refs \\
\hline$T / K$ & $\Delta G_{00 T^{*}}$ & $Q_{\mathrm{x}}{ }^{\mathrm{T}}$ & $Q_{Y}{ }^{0 \top}$ & $Q_{X Y}^{\top}$ & $\tau_{X(Y)}{ }^{\top(G)}$ & $\sigma_{Y(X)}{ }^{T(G)}$ & \\
\hline 265 & $70.2 \pm 0.3$ & $5.3 \pm 0.1$ & $-7.0 \pm 0.9$ & $1.3 \pm 0.4$ & 5.4 & -4.1 & b \\
\hline 281 & $72.5 \pm 0.3$ & $4.9 \pm 0.1$ & $-10 \pm 0.2$ & $1.4 \pm 0.5$ & 7.1 & -3.5 & b \\
\hline 298 & $74.8 \pm 0.5$ & $4.6 \pm 0.1$ & $-14 \pm 0.2$ & $1.7 \pm 0.7$ & 8.2 & -2.7 & $\mathrm{~b}$ \\
\hline$x$ & $\Delta G_{\times 00^{\neq}}$ & $Q_{Y}{ }^{X 0}$ & $Q_{T}{ }^{\mathrm{X} 0}$ & $Q_{Y T}{ }^{X}$ & $\sigma_{\mathrm{Y}(\mathrm{T})}{ }^{\mathrm{X}(\mathrm{G})}$ & $T_{(\mathrm{Y})}{ }^{\mathrm{X}(\mathrm{G})} / \mathrm{K}$ & \\
\hline $\mathrm{H}$ & $34 \pm 3$ & $45 \pm 9$ & $0.134 \pm 0.009$ & $-0.19 \pm 0.05$ & 0.70 & 237 & $\mathrm{~b}$ \\
\hline $4-\mathrm{NO}_{2}$ & $67 \pm 1$ & $39 \pm 3$ & $0.083 \pm 0.004$ & $-0.16 \pm 0.01$ & $0.52^{\mathrm{a}}$ & 244 & 16 \\
\hline $3-\mathrm{Br}-5-\mathrm{NO}_{2}$ & $83 \pm 1$ & $47 \pm 5$ & $0.036 \pm 0.003$ & $-0.18 \pm 0.02$ & $0.20^{a}$ & $261^{a}$ & 18 \\
\hline$Y$ & $\Delta G_{0 Y 0^{*}}$ & $Q_{x}{ }^{\mathrm{YO}}$ & $Q_{T}{ }^{O Y}$ & $Q_{X T}^{Y}$ & $\left.\tau_{X(T)}\right)^{Y(G)}$ & $T(\mathrm{X})^{\mathrm{Y}(\mathrm{G})} / \mathrm{K}$ & \\
\hline $4-\mathrm{OCH}_{3}$ & $23 \pm 8$ & $11 \pm 4$ & $0.19 \pm 0.05$ & $-0.021 \pm 0.007$ & 9.0 & 524 & $\mathrm{~b}$ \\
\hline $4-\mathrm{CH}_{3}$ & $26 \pm 3$ & $11 \pm 3$ & $0.17 \pm 0.03$ & $-0.021 \pm 0.005$ & 8.1 & 524 & b \\
\hline $\mathrm{H}$ & $31 \pm 8$ & $11 \pm 2$ & $0.14 \pm 0.03$ & $-0.023 \pm 0.009$ & 6.1 & 478 & b \\
\hline $4-\mathrm{Cl}$ & $45 \pm 4$ & $10 \pm 1$ & $0.09 \pm 0.01$ & $-0.018 \pm 0.004$ & 5.0 & 555 & $\mathrm{~b}$ \\
\hline
\end{tabular}
reactions of oxiranes $1 \mathbf{a}$-d with acids $2 a-e$ in the dioxane-1,2-dichloroethane mixture (7:3/v:v) at 265, 281, and $298 \mathrm{~K}$

${ }^{a}$ Experimentally observed IPP. $\quad \mathrm{b}$ This work.

The values of the cross-interaction coefficients $Q_{X Y}{ }^{\top}, Q_{Y T}{ }^{X}$, and $Q_{X T}{ }^{Y}$ indicates the influence of all types of the second-order interactions of the effects of the cross-varied factors on the free activation energy $\Delta G_{\mathrm{XYT}}{ }^{\neq}$. At the same time, the invariance of these coefficients when the parameters of fixed factors are changed indicates the absence of third-order interaction $\left(Q_{X Y T}=0\right)$. Twenty IPPS $\left(\tau_{X(Y)}{ }^{T(G)}, \tau_{X(T)}{ }^{Y(G)}, \sigma_{Y(X)}{ }^{T(G)}, \sigma_{Y(T)}{ }^{X(G)}, T_{(Y)}{ }^{X(G)}\right.$, and

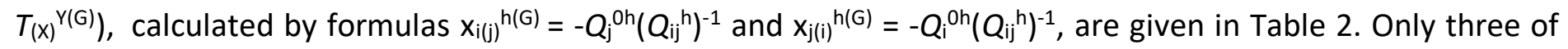
them turned out to be experimentally achievable. First of all it should be noted the IPP for the temperature $T_{(Y)}{ }^{X(G)}=261 \mathrm{~K}$ in the reactions of oxirane $\mathbf{1 d}$ with acids $\mathbf{2 a - 2} \mathbf{d}$, which is consistent with the above calculated value of the IPP $T_{(\mathrm{Y})}{ }^{\mathrm{X}}=262 \mathrm{~K}$. At this IPP, the free activation energy $\Delta G_{\mathrm{XYT}}{ }^{\neq \mathrm{IP}}$ should not depend on the effects of the substituents $Y$. The reason for the disappearance of the effects $Y$ on $\Delta G_{X Y T^{\neq}}$at the IPP $T_{(Y)}{ }^{X(G)}$ is the EECE:

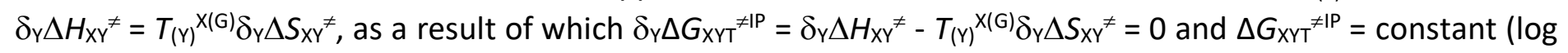
$k_{\mathrm{XYT}}=$ constant, $q_{\mathrm{Y}}{ }^{\mathrm{XT}}=0$ ). Because of the small difference between $T_{(\mathrm{Y})}{ }^{\mathrm{X}(\mathrm{G})}=261 \mathrm{~K}$ and temperature $265 \mathrm{~K}$ in the experiment, we have a rare opportunity to prove the physical reality of the enthalpy-entropy compensation phenomenon.

Due to the EECE in the reactions of oxirane $1 \mathbf{d}$ with acids $\mathbf{2 a}-\mathbf{d}$ at $265 \mathrm{~K}$, the substituents $\mathrm{Y}$ have no effect on the values of the free activation energy ${ }^{18}: \Delta G_{X Y T^{\neq}}(\mathrm{Y})=93.3(4-\mathrm{OMe}), 92.9(4-\mathrm{Me}), 93.0(\mathrm{H}), 93.1(4-\mathrm{Cl}) \mathrm{kJ}$ $\mathrm{mol}^{-1}$. Note that the IPP $T_{(\mathrm{Y})}{ }^{\mathrm{X}(\mathrm{G})}$, calculated from activation parameters (Equations 7,8 ), is called the 
compensation temperature $T_{\text {comp, }}$ while the term isokinetic temperature $T_{\text {iso }}$ refers to the IPP $T_{(\mathrm{Y})}{ }^{\mathrm{X}}$, calculated from kinetic data (Equations 3, 4). These points, calculated in different ways, practically coincide in magnitude.

Two IPPs for the constant of the substituent $Y \sigma_{Y(T)}{ }^{x(G)}=-Q_{T}{ }^{X 0}\left(Q_{Y T}{ }^{x}\right)^{-1}$, namely, $\sigma_{Y(T)}{ }^{x(G)}=0.52\left(X=4-N_{2}\right)$ and $\sigma_{\mathrm{Y}(\mathrm{T})}{ }^{\mathrm{X}(\mathrm{G})}=0.20\left(\mathrm{X}=3-\mathrm{Br}-5-\mathrm{NO}_{2}\right)$, were realized in the reactions of oxiranes $\mathbf{1 c}$ and $\mathbf{1 d}$ with acids $\mathbf{2 a}$-e at different temperatures. ${ }^{16,18}$ They fall in the experimental range of variation of $\sigma_{\gamma}=-0.27 \div 0.71$. A remarkable feature of these IPPs is that the free activation energy $\Delta G_{\mathrm{XYT}} \neq \mathrm{IP}$ at these points does not depend on temperature, that is possible if in the expression $\Delta G_{\mathrm{XY}}^{\neq \mathrm{IP}}=\Delta H_{\mathrm{XY}}^{\neq}-T \Delta S_{\mathrm{XY}}{ }^{\neq}$the activation entropy $\Delta S_{\mathrm{XY}}{ }^{\neq}=0$ and the free activation energy is determined only by the enthalpy term $\left.\left(\Delta G_{\mathrm{XYT}} \neq \mathrm{IP}=\Delta H_{\mathrm{XY}}\right)^{\neq}\right)$. In these reactions we were able not only to demonstrate a rare case of realization of two IPPs for the structural parameter $\sigma_{Y(T)}{ }^{X(G)}$, but also to realize unique transitions through these points, when the substituents $Y$ were varied. The reversal of the sign of the activation entropy $\Delta S_{\mathrm{XY}}{ }^{\neq}$after passing through these IPPs (Figure 1) leads to the inversion of the temperature effect on the free activation energy $\Delta G_{X Y T}{ }^{\neq}$. This unique situation is illustrated by Figure 2.

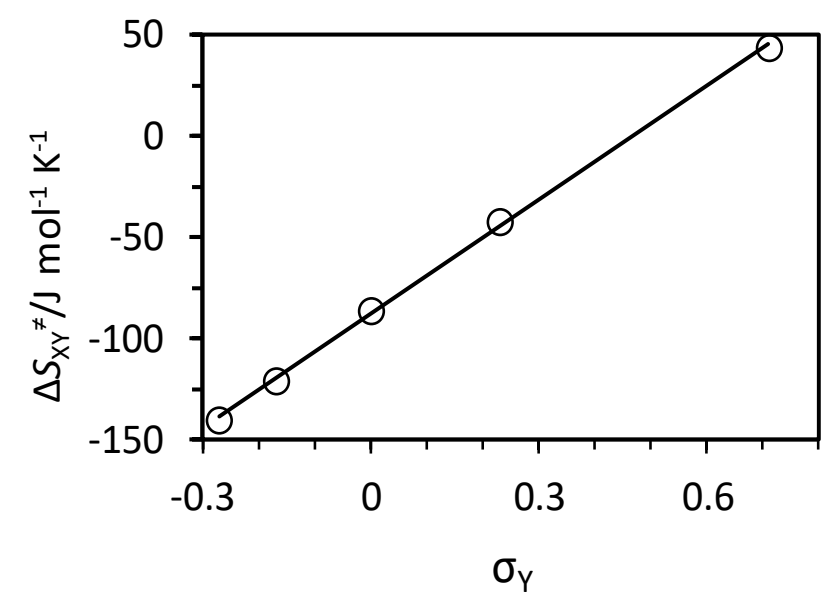

Figure 1. Sign inversion of the activation entropy $\Delta S_{X Y}{ }^{\neq}$after crossing the IPP $\sigma_{Y(T)}{ }^{X(G)}=0.52$ in reactions of oxirane $1 \mathrm{c}$ with acids $2 \mathrm{a}-\mathbf{e}$; the values of $\Delta S_{\mathrm{XY}}{ }^{\neq}$are taken from work. ${ }^{17}$

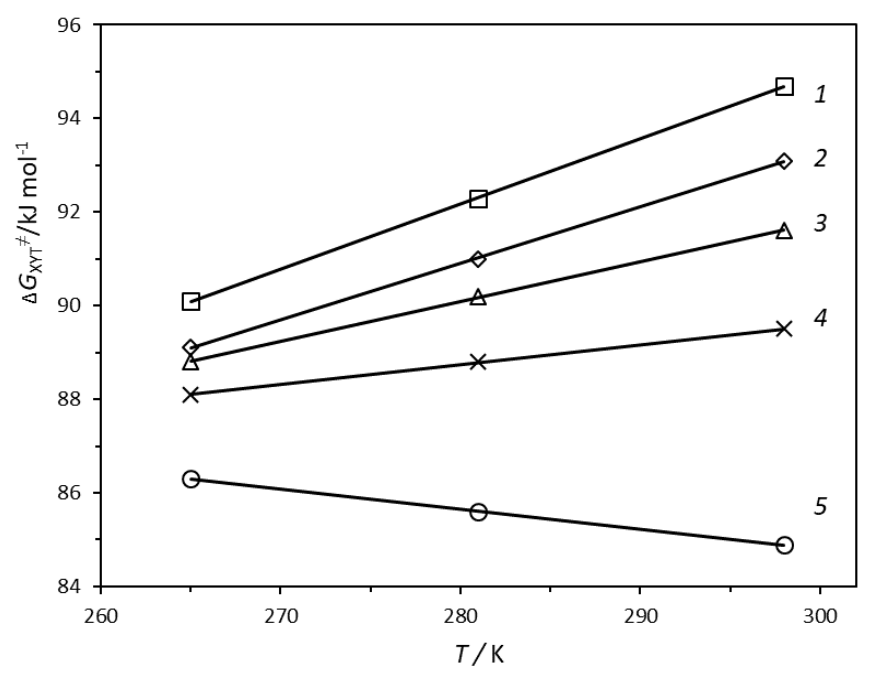

Figure 2. The reversal of the temperature effect on the free activation energy $\Delta G_{\mathrm{XYT}}{ }^{\neq}$after passing through the IPP $\sigma_{Y(T)}{ }^{X(G)}=0.52$ in reactions of oxirane $1 \mathbf{c}$ with acids $2 \mathbf{a}(1), \mathbf{b}(2), \mathbf{c}(3), \mathbf{d}(4)$, e (5); the values of $\Delta G_{X Y T^{*}}$ are taken from our previous work. ${ }^{17}$ 
Calculated IPPs can be used as quantitative criteria of the reaction mechanism presented in Scheme 2 . This mechanism provides for the formation of complex $\mathbf{A}$ with $\mathrm{H}$-bond between the oxirane and acid $\mathrm{HA}$ $\left(\mathrm{YC}_{6} \mathrm{H}_{4} \mathrm{SO}_{3} \mathrm{H}\right)$ in the first equilibrium stage. In the second and rate-determining stage, the substrate activated in this way undergoes a nucleophilic attack by the second acid molecules with the formation of transition state (TS) B. This stage proceeds according to the mechanism $A_{N} D_{N}$ with electrophilic assistance from the acid to the $\mathrm{C}-\mathrm{O}$ bond opening in the oxirane ring.

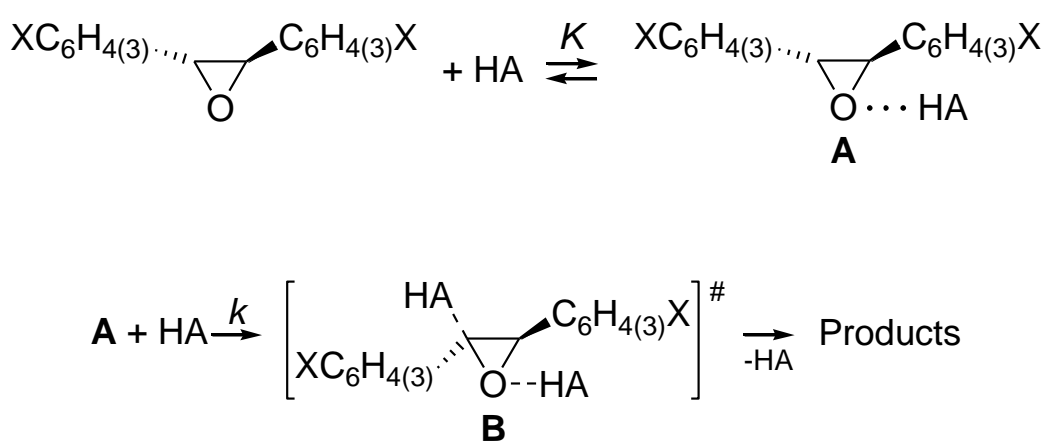

Scheme 2. Proposed mechanism of reactions of oxiranes 1a-d with arenesulfonic acids 2 a-e.

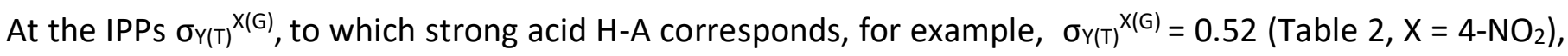
effective protonation of the oxirane occurs, so $\Delta \mathrm{S}_{1}<0$ in the first stage of the formation of $\mathrm{H}$-complex $\mathbf{A}$. The value for entropy of activation at the second stage $\Delta S_{2}{ }^{\neq}$depends on the character of the TS $B$, i.e., on the ratio

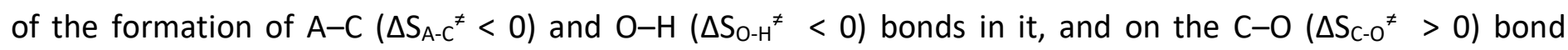

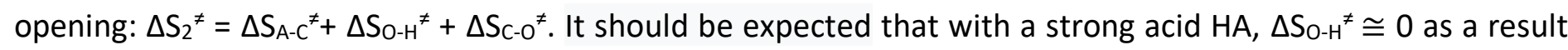
of the negligible change in the $\mathrm{O}-\mathrm{H}$ bond in TS $\mathbf{B}$ when compared to complex $\mathbf{A}$. In addition, TS B becomes $\mathrm{S}_{\mathrm{N}} 1-$ like ( $\mathrm{C}-\mathrm{O}$ bond opening dominates over $\mathrm{A}-\mathrm{C}$ bond formation) through the low nucleophilicity of $\mathrm{HA}$, as a result of which $\Delta \mathrm{S}_{\mathrm{C}-\mathrm{O}^{\neq}}>\left|\Delta \mathrm{S}_{\mathrm{A}-\mathrm{C}^{\mp}}\right|$ and $\Delta \mathrm{S}_{2}{ }^{\neq}>0$. At the IPP $\sigma_{\mathrm{Y}(\mathrm{T})}{ }^{\mathrm{X}(\mathrm{G})}=0.52$, the effective entropy of activation becomes zero $\left(S_{X Y}{ }^{\neq}=0\right)$, since $\Delta S_{2}{ }^{*}=\left|\Delta S_{1}\right|$. After transitioning through this IPP to more electron-acceptor substituents $Y$ with constant $\sigma_{Y}>0.52$, the $\mathrm{C}-\mathrm{O}$ bond opening in TS B grows. This leads to further growth in $\Delta \mathrm{S}_{2}{ }^{*}$, resulting in a situation where $\Delta S_{2}{ }^{\neq}>\left|\Delta S_{1}\right|$. The sign of the effective entropy of activation $\Delta S_{X Y^{*}}>0$ observed in Figure 1 is then reversed. This in turn causes an inversion of influence of the temperature effect on the free activation energy $\Delta G_{X Y T^{\ddagger}}$ (Figure 2).

According to the polylinearity principle the values of IPPs (Tables 1,2 ) obtained by $q_{i j}{ }^{h}$ interaction of the effects of two cross-varied factors $i$ and $j$ proved to be dependent linearly on the effects of a fixed factor $h$ :

$$
\begin{aligned}
& x_{i(j)}{ }^{h}=x_{i(j)}{ }^{0}+C_{h} X_{h} \\
& x_{i(j)}{ }^{h(G)}=x_{i(j)}{ }^{0(G)}+C_{h}{ }^{(G)} X_{h}
\end{aligned}
$$

The values of the coefficients of Equations 9 and 10 are given in Tables 3 and 4. Using them, we can quantitatively predict the conditions for the implementation of any IPP. Of particular interest is prediction of conditions under which experimental realization of IPPs for temperature, connected with the EECE, will be possible. For example, it follows from the data in Table 3 that the accessible IPPs $T_{(\mathrm{Y})}{ }^{\mathrm{X}}$, e. $\mathrm{g}$., $\left.T_{(\mathrm{Y})}\right)^{\mathrm{X}}=283 \mathrm{~K}$ or 303 $\mathrm{K}$, at which there is no influence of temperature on the effects of substituent $\mathrm{Y}\left(q_{\mathrm{Y}}^{\mathrm{XT}}=0\right)$, can be implemented if $\tau_{x}=7.11$ or 8.66, respectively. From coefficients of Equation 10 in Table 4 for the cross-reaction series in which the temperature $T$ and substituents $X$ are varied at a fixed substituent $Y$, it follows that easily accessible 
IPP $\left.\tau_{X(T)}\right)^{Y(G)}=0(X=H)$, at which there is no effect of temperature on the free activation energy $\left(\Delta S_{X Y}{ }^{\neq}=0\right)$, is realized if $\sigma_{Y}=0.80$, for instance, in the case of acid 2 with a substituent $Y$ such as 4-NO $\mathrm{NO}_{2}\left(\sigma_{Y}=0.78\right)$. In this way we can predict at a quantitative level the value of any IPP $x_{i(j)}{ }^{h}$ or $x_{i(j)}{ }^{h(G)}$ in two-factor cross-reaction series for a given value of the parameter of a fixed factor $h$. However, it should be borne in mind that the change of the fixed substituents $\mathrm{Y}$ has practically no effect on the values of the IPPs $T_{(\mathrm{X})}{ }^{\mathrm{Y}}$ (Tables 1,3$)$ and $T_{(\mathrm{X})}{ }^{\mathrm{Y}(\mathrm{G})}(\mathrm{Tables} 2,4)$.

Table 3. The coefficients of Equation 9 for the two-factor cross-reaction series

\begin{tabular}{|c|c|c|c|c|}
\hline Fixed factor $h$ & $x_{i(j)}{ }^{h}$ & $x_{i(j)}{ }^{0}$ & $C_{h} X_{h}$ & $r$ \\
\hline \multirow[t]{2}{*}{$T$} & $\tau_{X(Y)^{\top}}$ & $28.4 \pm 0.1$ & $(-6.27 \pm 0.04) 1000 / T$ & 0.999 \\
\hline & $\sigma_{Y(X)}{ }^{\top}$ & $3.5 \pm 1.5$ & $(-1.7 \pm 0.4) 1000 / T$ & 0.970 \\
\hline \multirow[t]{2}{*}{ Y } & $\tau_{X(T)}{ }^{Y}$ & $-3.4 \pm 0.3$ & $(-3.5 \pm 1.2) \sigma_{Y}$ & 0.900 \\
\hline & $10^{3} / T_{(x)}{ }^{Y}$ & $2.00 \pm 0.06$ & $(-0.5 \pm 0.3) \sigma_{Y}$ & - \\
\hline \multirow[t]{2}{*}{$x$} & $\sigma_{Y(T)}{ }^{X}$ & $-0.88 \pm 0.07$ & $(-0.27 \pm 0.02) \tau_{x}$ & 0.997 \\
\hline & $10^{3} / T_{(Y)}{ }^{\mathrm{X}}$ & $4.6 \pm 0.1$ & $(-0.15 \pm 0.04) \tau_{x}$ & 0.962 \\
\hline
\end{tabular}

Table 4. The coefficients of Equation 10 for the two-factor cross-reaction series

\begin{tabular}{|c|c|c|c|c|}
\hline Fixed factor $h$ & $x_{i(j)}{ }^{h(G)}$ & $X_{i(j)}{ }^{0(G)}$ & $c_{h}^{(G)} X_{h}$ & $r$ \\
\hline \multirow[t]{2}{*}{$T$} & $\tau_{X(Y)}{ }^{T(G)}$ & $-17 \pm 3$ & $(-0.08 \pm 0.01) T$ & 0.990 \\
\hline & $\left.\sigma_{Y(X)}\right)^{T(G)}$ & $-15.4 \pm 0.8$ & $(-0.042 \pm 0.003) T$ & 0.998 \\
\hline \multirow[t]{2}{*}{$Y$} & $\tau_{X(T)}{ }^{Y(G)}$ & $6.6 \pm 0.2$ & $(-8.2 \pm 1.1) \sigma_{Y}$ & 0.981 \\
\hline & $T(\mathrm{X})^{\mathrm{Y}(\mathrm{G})}$ & $522 \pm 19$ & $(46 \pm 96) \sigma_{Y}$ & - \\
\hline \multirow[t]{2}{*}{$x$} & $\left.\sigma_{Y(T)}\right)^{X(G)}$ & $0.73 \pm 0.16$ & $(0.10 \pm 0.05) \tau_{x}$ & 0.884 \\
\hline & $T_{(\mathrm{Y})} \mathrm{X( \textrm {G } )}$ & $235 \pm 9$ & $(4.5 \pm 2.8) \tau_{x}$ & 0.846 \\
\hline
\end{tabular}

\section{Three-parameter correlations}

To evaluate the total effects of substituents $X, Y$ and temperature $T$ on the rate of the investigated reactions considering all types of second-order interactions of the effects of the cross-varied factors, a PLE was used:

$$
\log k_{\mathrm{XYT}}=\log k_{000}+q_{\mathrm{X}}{ }^{00} \tau_{\mathrm{X}}+q_{\mathrm{Y}}{ }^{00} \sigma_{\mathrm{Y}}+q_{\mathrm{T}}^{00} \mathrm{t}_{\mathrm{K}}+q_{\mathrm{XY}}{ }^{0} \tau_{\mathrm{X}} \sigma_{\mathrm{Y}}+q_{\mathrm{XT}}{ }^{0} \tau_{\mathrm{X}} \mathrm{t}_{\mathrm{K}}+q_{\mathrm{YT}}{ }^{0} \sigma_{\mathrm{Y}} \mathrm{t}_{\mathrm{K}}
$$

Here $t_{K}(T)=(1 / T-1 / 281) \times 10^{3}=0.215(265 \mathrm{~K}), 0(281 \mathrm{~K})$, and $-0.203(298 \mathrm{~K})$ is an operationally introduced internal measure for temperature factor; $k_{000}$ is the rate constant under standard conditions $\left(\tau_{X}=\sigma_{Y}=t_{K}=0\right)$, $q_{X}{ }^{00}, q_{Y}{ }^{00}$ and $q_{T}{ }^{00}$ are the sensitivity coefficients of standard reactions $\left(\sigma_{Y}=t_{K}=0, \tau_{X}=t_{K}=0\right.$, and $\tau_{X}=\sigma_{Y}=0$, respectively); $q_{\mathrm{XY}}{ }^{0}, q_{\mathrm{XT}}{ }^{0}, q_{\mathrm{YT}}{ }^{0}$ are the coefficients of the second-order interactions under standard conditions ( $\mathrm{t}_{\mathrm{K}}$ $=0, \sigma_{Y}=0$, and $\tau_{X}=0$, respectively). The treatment of the results of a multifactorial kinetic experiment ${ }^{17}$ by Equation 11 gave the following multilinear regression (MLR), adequately describing the reactivity of the investigated system (hereinafter, $S D$ is the standard deviation, $N$ are experimental data points, $F$ is the Fisher criterion):

$$
\begin{aligned}
& \log k_{\mathrm{XYT}}=(-0.83 \pm 0.02)+(-0.886 \pm 0.007) \tau_{\mathrm{X}}+(2.0 \pm 0.1) \sigma_{\mathrm{Y}}+(-1.8 \pm 0.1) \mathrm{t}_{\mathrm{K}}+ \\
& (-0.32 \pm 0.03) \tau_{\mathrm{X}} \sigma_{\mathrm{Y}}+(-0.55 \pm 0.04) \tau_{\mathrm{X}} \mathrm{t}_{\mathrm{K}}+(-2.1 \pm 0.2) \sigma_{\mathrm{Y}} t_{\mathrm{K}} \\
& R=0.998, S D=0.077, N=43, F=3173
\end{aligned}
$$


In MLR 12 the values of all coefficients are statistically significant and agree with those calculated in partial correlations at standard conditions (see in Table 1 the values of log $k_{00 \mathrm{~T}}, q_{\mathrm{X}}{ }^{0 \top}, q_{\mathrm{Y}}{ }^{0 \top}, q_{\mathrm{XY}}{ }^{\top}(T=281 \mathrm{~K}) ; q_{\mathrm{T}}{ }^{\mathrm{X0}}(\mathrm{X}=\mathrm{H})$, $\left.q_{T}{ }^{\mathrm{OY}}(\mathrm{Y}=\mathrm{H}), q_{\mathrm{XT}}{ }^{\mathrm{Y}}(\mathrm{Y}=\mathrm{H}), q_{\mathrm{YT}}{ }^{\mathrm{X}}(\mathrm{X}=\mathrm{H})\right)$.

The combined effects of substituents $X, Y$ and $T$ on the free activation energy $\Delta G_{X Y T^{*}}$ were evaluated by a PLE:

$$
\Delta G_{X Y T}{ }^{\neq}=\Delta G_{000}{ }^{\neq}+Q_{X}{ }^{00} \tau_{X}+Q_{Y}{ }^{00} \sigma_{Y}+Q_{T}{ }^{00} t_{G}+Q_{X Y}{ }^{0} \tau_{X} \sigma_{Y}+Q_{X T}{ }^{0} \tau_{X} t_{G}+Q_{Y T}{ }^{0} \sigma_{Y} t_{G}
$$

Here $t_{G}(T)=T-281=-16(265 \mathrm{~K}), 0(281 \mathrm{~K}), 17(298 \mathrm{~K})$ is an internal measure for the temperature factor; $\Delta G_{000}{ }^{\neq}$is the free activation energy at $\tau_{X}=\sigma_{Y}=t_{G}=0 ; Q_{X}{ }^{00}, Q_{Y}{ }^{00}, Q_{T}{ }^{00}$ are the sensitivity coefficients under standard conditions $\left(\sigma_{Y}=\mathrm{t}_{\mathrm{G}}=0, \tau_{\mathrm{X}}=\mathrm{t}_{\mathrm{G}}=0, \tau_{\mathrm{X}}=\sigma_{\mathrm{Y}}=0\right.$, respectively); $Q_{\mathrm{XY}}{ }^{0}, Q_{\mathrm{XT}}{ }^{0}, Q_{\mathrm{YT}}{ }^{0}$ are the coefficients of the second-order interactions under standard conditions $\left(t_{G}=0, \sigma_{Y}=0, \tau_{X}=0\right.$, respectively). When calculating the coefficients of PLE 13 using the values $\Delta G_{X Y T}{ }^{\neq}$collected in work ${ }^{17}$, the following MLR was obtained:

$$
\begin{aligned}
& \Delta G_{X Y T^{*}}=(72.4 \pm 0.2)+(4.93 \pm 0.07) \tau_{X}+(-10 \pm 1) \sigma_{Y}+(0.12 \pm 0.02) t_{G}+ \\
& (1.3 \pm 0.3) \tau_{X} \sigma_{Y}+(-0.016 \pm 0.005) \tau_{X} t_{G}+(-0.15 \pm 0.03) \sigma_{Y} t_{G} \\
& R=0.994, S D=0.773, N=39, F=871
\end{aligned}
$$

All coefficients of MLE 14 agree within the accuracy limits of their determination with those calculated in partial correlations at standard conditions (see in Table 2 the values of $\Delta G_{00 T^{*}}{ }, Q_{\mathrm{X}}{ }^{0 \mathrm{~T}}, Q_{Y}{ }^{0 \top}, Q_{\mathrm{XY}}{ }^{\top}(T=281 \mathrm{~K}) ; Q_{\mathrm{T}}{ }^{\mathrm{XO}}$ $\left.(X=H), Q_{T}{ }^{\mathrm{OY}}(\mathrm{Y}=\mathrm{H}), Q_{X T}{ }^{\mathrm{Y}}(\mathrm{Y}=\mathrm{H}), Q_{Y T}{ }^{\mathrm{X}}(\mathrm{X}=\mathrm{H})\right)$.

The isoparametricity considered above is an inherent property of two-parameter relationships with cross terms. The three-parameter relationships like PLEs 11 and 13 as well as MLRs 12 and 14 exhibit other striking properties. Each of them is characterized by the critical values (CVs) of the parameters of the variable factors, at which some of their terms vanish. Consider, for example, PLE 13 in the following forms:

$$
\begin{aligned}
& \Delta G_{\mathrm{XYT}}{ }^{\neq}=\Delta G_{000^{\neq}}+\left(Q_{\mathrm{X}}^{00}+Q_{\mathrm{XY}}^{0} \sigma_{\mathrm{Y}}\right) \tau_{\mathrm{X}}+\left(Q_{\mathrm{Y}^{00}}+Q_{\mathrm{YT}}{ }^{0} \mathrm{t}_{\mathrm{G}}\right) \sigma_{\mathrm{Y}}+\left(Q_{\mathrm{T}}{ }^{00}+Q_{\mathrm{XT}}{ }^{0} \tau_{\mathrm{X}}\right) \mathrm{t}_{\mathrm{G}} \\
& \Delta G_{X Y T}{ }^{\neq}=\Delta G_{000^{\neq}}+\left(Q_{X}{ }^{00}+Q_{X T}{ }^{0} t_{G}\right) \tau_{X}+\left(Q_{Y}{ }^{0}+Q_{X Y}{ }^{0} \tau_{X}\right) \sigma_{Y}+\left(Q_{T}{ }^{00}+Q_{Y T}{ }^{0} \sigma_{Y}\right) t_{G}
\end{aligned}
$$

Now we can easily determine three pairs of the CVs for which the corresponding expressions in the brackets become zero: $\sigma_{Y(X)}{ }^{\mathrm{CV}}=-Q_{X}{ }^{00}\left(Q_{X Y}{ }^{0}\right)^{-1}$ and $\sigma_{Y(T)}{ }^{C V}=-Q_{T}{ }^{00}\left(Q_{Y T}{ }^{0}\right)^{-1}, \tau_{X(Y)}{ }^{C V}=-Q_{Y}{ }^{00}\left(Q_{X Y}{ }^{0}\right)^{-1}$ and $\tau_{X(T)}{ }^{C V}=-$ $Q_{\mathrm{T}}{ }^{00}\left(Q_{X \mathrm{X}}{ }^{0}\right)^{-1}, \mathrm{t}_{\mathrm{G}(\mathrm{Y})}{ }^{\mathrm{CV}}=-Q_{Y}{ }^{00}\left(Q_{\mathrm{YT}^{0}}\right)^{-1}$ and $\mathrm{t}_{\mathrm{G}(\mathrm{X})}{ }^{\mathrm{CV}}=-Q_{\mathrm{X}}{ }^{00}\left(Q_{\mathrm{XT}}{ }^{0}\right)^{-1}$. In this context, the following CVs are the attributes of MLR 14 chosen as an example: $\sigma_{Y(X)}{ }^{C V}=-3.79$ and $\sigma_{Y(T)}{ }^{C V}=0.80, \tau_{X(Y)}{ }^{C V}=7.69$ and $\tau_{X(T)}{ }^{C V}=7.50, t_{G(Y)}{ }^{C V}=-66.7$ $(T=214 \mathrm{~K})$ and $\mathrm{t}_{\mathrm{G}(\mathrm{X})}{ }^{\mathrm{CV}}=308(T=589 \mathrm{~K})$. In the represented series of CVs the following parts of MLR 14 disappear: $(-10 \pm 1) \sigma_{Y}+(1.3 \pm 0.3) \tau_{X} \sigma_{Y}$ at $\tau_{X(Y)}{ }^{C V},(4.93 \pm 0.07) \tau_{X}+(1.3 \pm 0.3) \tau_{X} \sigma_{Y}$ at $\sigma_{Y(X)}{ }^{C V},(0.12 \pm 0.02) t_{G}+(-$ $0.016 \pm 0.005) \tau_{x} t_{G}$ at $\tau_{X(T)}{ }^{C V},(4.93 \pm 0.07) \tau_{x}+(-0.016 \pm 0.005) \tau_{x} t_{G}$ at $t_{G(X)}{ }^{C V},(0.12 \pm 0.02) t_{G}+(-0.15 \pm 0.03) \sigma_{Y} t_{G}$ at $\sigma_{\mathrm{Y}(\mathrm{T})}{ }^{\mathrm{CV}}$, and $(-10 \pm 1) \sigma_{\mathrm{Y}}+(-0.15 \pm 0.03) \sigma_{\mathrm{Y}} \mathrm{t}_{\mathrm{G}}$ at $\mathrm{t}_{\mathrm{G}(\mathrm{Y})}{ }^{\mathrm{CV}}$. None of these CVs have been implemented under experimental conditions. The same applies to the MLR 12. It should be noted that experimental evidence for the realization of numerous CVs of the parameters of the variable factors (structure and temperature) was obtained in nucleophilic substitution reactions at benzyl carbon atom. ${ }^{10,11}$ 


\section{Conclusions}

In this work we have shown that oxirane ring opening reactions are suitable for investigation of the isoparametric properties of the cross-reaction series and especially such an important aspect of

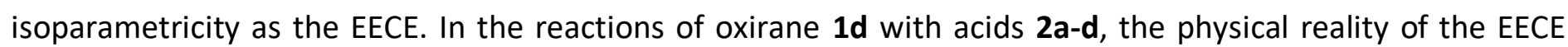
was proved: at a temperature of $265 \mathrm{~K}$ in an experiment, close to the IPP $T_{(\mathrm{Y})}{ }^{\mathrm{X}}=262 \mathrm{~K}\left(T_{(\mathrm{Y})}{ }^{\mathrm{X}(\mathrm{G})}=261 \mathrm{~K}\right)$, the influence of substituents $Y$ in the acid reagent on the rate (free activation energy) of these reactions disappears. In all, five of the forty calculated IPPs (Tables 1 and 2) were implemented in the two-factor crossreaction series of the structure-structure and structure-temperature types, and two of them $\left(\sigma_{Y(T)}\right)^{X(G)}=0.20$ and $\sigma_{Y(T)}{ }^{X(G)}=0.52$ ) were passed through with the demonstration of the isoparametricity paradox. Linear dependencies of the values of IPPs, obtained by interaction of mutual effects of two variable factors, on the effects of the third fixed factor has proved to be valuable in prediction at a quantitative level the conditions for the implementation of any IPP in cross-reaction series. Important information was also obtained about the intriguing properties of the three-parameter relationships, concerning such their attributes as the CVs of the parameters of the variable factors. At a CV corresponding terms of these relationships disappear.

Knowledge of these poorly studied latent properties of cross-reaction series expands our notions on quantitative regularities of organic reactivity. In this context, new interesting discoveries should be expected with further study of the cross effects of structure, catalyst, temperature, medium and other internal and external factors on the rate and activation parameters of ring opening reactions of aromatic substituted derivatives of small heterocycles such as oxiranes, thiiranes, aziridines and others.

\section{Experimental Section}

The third order rate constants $k_{\mathrm{XYT}} \mathrm{M}^{-2} \mathrm{~s}^{-1}$ (the first order with respect to the oxirane substrate and second order with respect to the acidic reagent), previously obtained ${ }^{17}$ for the reactions of oxiranes 1 a-d with acids 2a-e at different temperatures (Scheme) as well as the values of the free activation energy $\Delta G_{\mathrm{XYT}}{ }^{\neq}$of these reactions were used to calculate two-and three-factor regressions with cross-terms in accordance with the PLEs $2-4$ and 11 for kinetic data and PLEs $6-8$ and 13 for activation data. The coefficients of PLEs $2-4$ and 6 - 8 given accordingly in Tables 1 , and 2 were then used to calculate the values of IPPs for the parameters of variable factors (substituents $\mathrm{X}, \mathrm{Y}$ and temperature $T$ ) in each considered two-factor reaction series. The coefficients of MLRs 12 and 14 taking into account all variants of the second-order interactions of the effects of three factors were used to calculate the CVs for each of them.

The accuracy of the correlation parameters was estimated in terms of the standard deviation SD, which was derived by the statistical method from $N$ experimental data points. Statistical data processing was carried out for the confidence level of 0.95 .

\section{Acknowledgements}

The study was carried out within the Fundamental Research Programme funded by the Ministry of Education and Science of Ukraine (Project No. 0116U002519) 


\section{Supplementary Material}

Kinetic and activation parameters of reactions between oxiranes $\mathbf{1 a - d}$ and arenesulfonic acids $\mathbf{2 a - e}$ are available in the Supplementary File.

\section{References}

1. Wells, P. R. Chem. Rev. 1963, 63, 171. https://doi.org/10.1021/cr60222a005

2. Chapman, N.; Shorter, J. Eds. Advances in Linear Free Energy Relationships, Plenum Press: New York, 1972. https://doi.org/10.1007/978-1-4615-8660-9

3. Johnson, C. D. The Hammett Equation, Cambridge University Press: Cambridge, 1973.

4. Chapman, N.; Shorter, J. Eds. Correlation Analysis: Recent Advances, Plenum Press: New York, 1978. https://doi.org/10.1007/978-1-4615-8831-3

5. Shorter, J. Correlation Analysis of Organic Reactivity, with Particular Reference to Multiple Regression, John Wiley \& Sons Inc.: Somerset, NJ, 1982.

6. Williams, A. Free Energy Relationships in Organic and Bioorganic Chemistry, RSC: Cambridge, 2003. http://doi.org/10.1039/9781847550927

7. Palm, V. A. Bases of Quantitative Theory of Organic Reactions [in Russian], Khimiya: Leningrad, 1977.

8. Palm, V. A.; Istomin, B. I. Reakts. Sposonost Org. Soedin. (Tartu). 1969, 6, 427.

9. Shpan'ko, I. V. Mendeleev Commun. 1991, 1, 119. https://doi.org/10.1070/MC1991v001n04ABEH000073

10. Shpanko, I. V.; Kim, S. I.; Koh, H. J.; Lee, I. Bull. Korean Chem. Soc. 1995, 16, 533.

11. Shpan'ko, I.V. Theoret. Experim. Chem. 1999, 35, 63. https://doi.org/10.1007/BF02511491

12. Shpan'ko, I. V. Theoret. Experim. Chem. 2001, 37, 265. https://doi.org/10.1023/A:1013879231709

13. Shpan'ko, I. V.; Sadovaya, I. V. Theoret. Experim. Chem. 2010, 46, 176. https://doi.org/10.1007/s11237-010-9136-z

14. Shpan'ko, I. V.; Sadovaya, I. V. Kinetics Catalysis 2014, 55, 56. https://doi.org/10.1134/S002315841401011X

15. Shpan'ko, I. V.; Sadovaya, I.V. Russ. J. Gen. Chem. 2016, 86, 2418. https://doi.org/10.1134/S1070363216110037

16. Shpan'ko, I. V.; Sadovaya, I. V. Russ. J. Phys. Chem. A 2016, 90, 2332. https://doi.org/10.1134/S0036024416120268

17. Shpan'ko I.V.; Sadovaya, I. V. Russ. J. Gen. Chem. 2017, 87, 2552. https://doi.org/10.1134/S107036321711007X

18. Shpan'ko, I. V.; Sadovaya, I.V. Reac. Kinet. Mech. Cat. 2018, 123, 473. https://doi.org/10.1007/s11144-017-1340-6

19. Exner, O. Progr. Phys. Org. Chem. 1973, 10, 411. https://doi.org/10.1002/9780470171899.ch6

20. Krug, R. R.; Hunter, W. G.; Grieger, R. A. Nature 1976, 261, 566. 
https://doi.org/10.1038/261566a0

21. Linert, W.; Jameson, R. F. Chem. Soc. Rev. 1989, 18, 477. https://doi.org/10.1039/CS9891800477

22. Linert, W. Chem. Soc. Rev. 1994, 23, 429. https://doi.org/10.1039/CS9942300429

23. McBane, G. C. J. Chem. Educ. 1998, 75, 919. https://doi.org/10.1021/ed075p919

24. Liu, L.; Guo Q.-X. Chem. Rev. 2001, 101, 673. https://doi.org/10.1021/cr990416z

25. Sharp, K. Protein Sci. 2001, 10, 661. https://doi.org/10.1110/ps.37801

26. Cornish-Bowden, A. J. Biosci. 2002, 27, 121. https://doi.org/10.1007/BF02703768

27. Starikov, E. B.; Norden, B. J. Phys. Chem. B 2007, 111, 14431. https://doi.org/10.1021/ip075784i

28. Norwisz, J.; Musielak, T. J. Therm. Anal. Calorim. 2007, 88, 751. https://doi.org/10.1007/s10973-006-8139-4

29. Perez-Benito, J. F.; Mulero-Raichs, M. J. Phys. Chem. A 2016, 120, 7598. https://doi.org/10.1021/acs.jpca.6b08079

30. Pan, A.; Biswas, T.; Rakshit, A. K.; Moulik, S. P. J. Phys. Chem. B 2015, 119, 15876. https://doi.org/10.1021/acs.jpcb.5b09925

31. Pan, A.; Kar, T.; Rakshit, A. K.; Moulik, S. P. .J. Phys. Chem. B 2016, 120, 10531. https://doi.org/10.1021/acs.jpcb.6b05890

32. Parmon, V. N. Reac. Kinet. Mech. Cat. 2016, 118, 165. https://doi.org/10.1007/s11144-016-1005-x

33. Cornish-Bowden, A. J. Biosci. 2017, 42, 665. https://doi.org/10.1007/s12038-017-9719-0 